\title{
Second-order hyperbolic s.p.d.e.'s driven by boundary noises
}

\author{
Robert C. Dalang and Olivier Lévêque
}

\begin{abstract}
We study a class of second-order linear hyperbolic partial differential equations in spatial dimension $d$, driven by spatially correlated Gaussian noise that is white in time and concentrated in space on a hypersurface. For the case of isotropic Gaussian noise concentrated on a sphere, we give an explicit necessary and sufficient condition on the spatial covariance of the noise which guarantees that the solution of the equation is a function-valued process indexed by time. In the case of spatially homogeneous noise concentrated on a hyperplane $H$, we provide a necessary and sufficient condition for existence of a function-valued solution, defined everywhere outside of $H$, as well as a (different) necessary and sufficient condition for existence of a real-valued process solution, defined for all times and in all of space (including on $H$ ). A sufficient condition for Hölder continuity is provided, and existence and uniqueness for a non-linear form of the equation is established.
\end{abstract}

\section{Introduction}

This paper surveys some recent developments in the study of stochastic partial differential equations (s.p.d.e.'s) driven by boundary noises, as developed in $[10,11,16]$. We shall present the main results, along with some ideas and intuitions concerning the proofs, and point out other relevant results in the literature.

S.p.d.e.'s are partial differential equations with random source terms, which are strongly irregular, both in time and space. They provide models for physical phenomena with temporal and spatial variations that are too rapid to be well described by deterministic models. Examples of such phenomena are to be found in various domains, such as oceanography $[1,6]$, fluid mechanics [5] or mathematical finance [3].

There are several approaches to the study of s.p.d.e.'s. We follow mainly the approach of J. B. Walsh [28], who considers partial differential equations driven by additive noises (mainly space-time white noise). Solutions of such equations are described as random fields indexed by the time and space variables, and are expressed as generalized stochastic integrals with respect to a martingale measure constructed from the noise under consideration. A different approach is described in $[13,14]$, where solutions are processes indexed by the time variable with values in some functional space over the space variable, namely Banach or Hilbert spaces. We will also use some aspects of this approach.

Our focus is second order hyperbolic partial differential equations driven by boundary noises. A typical example is the equation that describes wave propagation in ordinary three-dimensional space perturbed by a noise concentrated on a surface. This type of situation might for example arise in the study of the sound wave produced by the noise of rain falling on the surface of a lake. This noise is composed of a large number of small contributions (namely the droplets of rain); on a large scale, it is therefore natural to consider that it is Gaussian. Moreover, it is concentrated on a surface (namely the lake surface), so the pressure wave emitted by this noise satisfies (in a first order approximation) a wave equation driven by an additive noise source concentrated on the surface of the lake.

There is a growing literature on partial differential equations driven by boundary noises, though the noise is generally considered as a stochastic boundary condition. However, most studies concern either the case where the space dimension is equal to one (see $[12,17])$, or the case where the equation is parabolic $($ see $[18,27])$. Here, we consider hyperboblic equations in higher dimensional space, which presents specific difficulties because of the singularity of the Green kernel of such equations.

Our generic equation will be of the form

$$
L u(t, x)=\dot{F}(t, x) \delta_{S}(x), \quad(t, x) \in \mathbb{R}_{+} \times \mathbb{R}^{d},
$$

where $L$ is a second order linear hyperbolic operator given by

$$
L=\frac{\partial^{2}}{\partial t^{2}}+2 a \frac{\partial}{\partial t}+b-\Delta, \quad \text { with } a, b \in \mathbb{R},
$$

$\left\{\dot{F}(t, x),(t, x) \in \mathbb{R}_{+} \times S\right\}$ is a generalized Gaussian process and $\delta_{S}$ is the Dirac measure on some hypersurface $S$ in $\mathbb{R}^{d}$.

Since this equation is linear, it always has a solution in the space of Schwartz distributions (which satisfies a weak formulation of the equation), that is formally given by

$$
u(t, x)=\int_{0}^{t} d s \int_{S} d \sigma(y) \dot{F}(s, y) G(t-s, x-y),
$$

where $\sigma$ is the uniform measure on $S$ and $G$ is the Green kernel of the operator $L$. Note that the above formal expression needs to be rigorously defined as the stochastic integral of a deterministic (Schwartz) distribution $G$ with respect to a martingale-measure constructed from the noise $\dot{F}$ (the details are carried out in $[8,16])$. Under mild assumptions on the covariance of the noise, it is possible to show that for each $t \in \mathbb{R}_{+}, u(t \cdot \cdot)$ belongs to a space of distributions on $\mathbb{R}^{d}$. 
- if we want to study related non-linear models, but wish to avoid defining the non-linear transformation of a Schwartz distribution, we also need $u$ to be function-valued.

In the following, we focus on two particularly simple examples of surfaces: the (hyper)sphere and the hyperplane in $\mathbb{R}^{d}$. As we will see, the results obtained in both cases show significant similarities. The noise will be concentrated on one of these surfaces, and within the surface, will exhibit sufficient symmetry, such as isotropy or spatial homogeneity. In this way, it remains possible to use Fourier analysis techniques developed for hyperbolic s.p.d.e.'s driven by spatially homogeneous noises (see the references mentioned above).

\section{Isotropic Gaussian noise on a sphere}

For simplicity, we will consider here equation (1.1) in the spatial domain $D=B(0,1) \subset \mathbb{R}^{d}$, driven by noise concentrated on the sphere $S=\partial D$. This equation, together with vanishing initial conditions and Neumann boundary conditions, is expressed as follows:

$$
\begin{cases}L u(t, x)=\dot{F}(t, x) \delta_{S}(x), & (t, x) \in \mathbb{R}_{+} \times D, \\ u(0, x)=\frac{\partial u}{\partial t}(0, x)=0, & x \in D, \\ \frac{\partial u}{\partial \nu}(t, x)=0, & (t, x) \in \mathbb{R}_{+} \times S,\end{cases}
$$

where $L$ is the operator defined in (1.2) and $\frac{\partial u}{\partial \nu}$ is the normal derivative of $u$ at the boundary. It is possible to show that the weak formulation of this equation (see [10] for more details) is the same as that of the equation

$$
\begin{cases}L u(t, x)=0, & (t, x) \in \mathbb{R}_{+} \times D, \\ u(0, x)=\frac{\partial u}{\partial t}(0, x)=0, & x \in D, \\ \frac{\partial u}{\partial \nu}(t, x)=\dot{F}(t, x), & (t, x) \in \mathbb{R}_{+} \times S,\end{cases}
$$

so that the boundary noise can also be interpreted as a stochastic boundary condition.

In any case, the weak solution is given by the formal expression

$$
u(t, x)=\int_{0}^{t} d s \int_{S} d \sigma(y) \dot{F}(s, y) G(t-s, x, y), \quad(t, x) \in \mathbb{R}_{+} \times D,
$$

where $G$ is the Green kernel of the operator $L$ in the domain $D$.

We now specify the precise assumptions to be made on the noise $\dot{F}$. As mentioned above, the process $\left\{\dot{F}(t, x),(t, x) \in \mathbb{R}_{+} \times S\right\}$ is assumed to be a generalized centered Gaussian process with covariance

$$
\mathbb{E}(\dot{F}(t, x) \dot{F}(s, y))=\delta_{0}(t-s) g(x, y),
$$

where $\delta_{0}$ is the Dirac measure at 0 and $g$ is a symmetric non-negative definite distribution on $S^{d} \times S^{d}$ (which needs therefore not necessarily be a function). Our objective is to establish the minimal condition on $g$ which guarantees that $u$ is a function-valued process. For this, we need the classical spectral theorem which states that there exists an orthonormal basis $\left\{e_{n}, n \in \mathbb{N}\right\}$ of $L^{2}(D)$ and non-negative numbers $\left\{\lambda_{n}, n \in \mathbb{N}\right\}$ tending to infinity as $n \rightarrow \infty$ such that

$$
\Delta e_{n}(x)+\lambda_{n} e_{n}(x)=0, \quad x \in D, \quad \text { and } \quad \frac{\partial e_{n}}{\partial \nu}(x)=0, \quad x \in S
$$

Let us now define the coefficients

$$
\gamma_{n}=\int_{S} d \sigma(x) \int_{S} d \sigma(y) e_{n}(x) g(x, y) e_{n}(y)
$$

needed to state the first result.

Theorem 2.1. The unique weak solution $u$ of equation (2.1) (or equivalently, equation (2.2)) is such that $u(t, \cdot) \in L^{2}(D)$ for all $t \in \mathbb{R}_{+}$if and only if

$$
\sum_{n \in \mathbb{N}} \frac{\gamma_{n}}{1+\lambda_{n}}<\infty
$$


Proof. We give a (formal) sketch of the proof, since details can be found in [10]. Assume for simplicity that $a=b=0$. The Green kernel of the operator $L$ can be decomposed in the basis $\left\{e_{n}\right\}$ as follows:

$$
G(t, x, y)=\sum_{n \in \mathbb{N}} G_{n}(t) e_{n}(x) e_{n}(y)
$$

where the $G_{n}(t)$ are given by

$$
G_{n}(t)=\frac{\sin \left(\sqrt{\lambda_{n}} t\right)}{\sqrt{\lambda_{n}}}
$$

We can therefore rewrite $u$ formally as

$$
u(t, x)=\sum_{n \in \mathbb{N}} \int_{0}^{t} d s \int_{S} d \sigma(y) \dot{F}(s, y) G_{n}(s) e_{n}(y) e_{n}(x),
$$

and a formal use of formulas (2.3), (2.4) and Fubini's theorem yields

$$
\mathbb{E}\left(\int_{D} d x u(t, x)^{2}\right)=\sum_{n \in \mathbb{N}} \gamma_{n} \int_{0}^{t} d s G_{n}(s)^{2} .
$$

It is straightforward to check that for fixed $t \in \mathbb{R}_{+}$,

from which the result follows.

$$
\int_{0}^{t} d s G_{n}(s)^{2} \underset{n \rightarrow \infty}{\sim} \frac{1}{1+\lambda_{n}},
$$

While condition (2.5) is necessary and sufficient, it is not as explicit as we would like. For instance, it is not immediately clear whether space-time white noise on the sphere satisfies this condition or not. So we would like to translate this condition into a more explicit form. For this, we need the following supplementary assumption on the noise : we assume that the covariance $g$ is isotropic, that is, that there exists some function $f$ on $[-1,+1]$ such that $g(x, y)=f(x \cdot y)$ (in a distributional sense), where $x \cdot y$ represents the Euclidean inner product of $x$ and $y$ in $\mathbb{R}^{d}$.

If the covariance function $f$ is continuous, then it must have a very special form, given by Schönberg's theorem [25].

Theorem 2.2. Let $f:[-1,+1] \rightarrow \mathbb{R}$ be a continuous function. Then $f$ is non-negative definite on $S$, that is,

$$
\sum_{i, j=1}^{n} c_{i} c_{j} f\left(x_{i} \cdot x_{j}\right) \geq 0, \quad \text { for all } n \geq 1, c_{1}, \ldots, c_{n} \in \mathbb{R}, x_{1}, \ldots, x_{n} \in S,
$$

if and only if there exists non-negative numbers $\left\{a_{l}, l \in \mathbb{N}\right\}$ such that

$$
f(t)=\sum_{l \in \mathbb{N}} a_{l} P_{l}(d, t), \quad t \in[-1,+1], \quad \text { and } \quad \sum_{l \in \mathbb{N}} a_{l}<\infty,
$$

where the $P_{l}(d, \cdot)$ are the (generalized) Legendre polynomials defined by

$$
P_{l}(d, t)=\left(-\frac{1}{2}\right)^{l} \frac{\Gamma\left(\frac{d-1}{2}\right)}{\Gamma\left(l+\frac{d-1}{2}\right)}\left(1-t^{2}\right)^{\frac{3-d}{2}}\left(\frac{d}{d t}\right)^{l}\left(1-t^{2}\right)^{l+\frac{d-3}{2}}, \quad l \in \mathbb{N}, t \in[-1,+1],
$$

and $\Gamma$ is the Euler Gamma function.

In order to have at hand a more general class of isotropic covariances $f$ which need not be continuous functions, we can simply replace (2.6) by the weaker condition that there exists some $r>0$ such that

$$
f(t)=\sum_{l \in \mathbb{N}} a_{l} P_{l}(d, t), \quad t \in[-1,+1], \quad \text { and } \quad \sum_{l \in \mathbb{N}} \frac{a_{l}}{(1+l)^{r}}<\infty .
$$

The following theorem is valid for any space dimension $d \geq 2$.

Theorem 2.3. Equation (2.1) (or equivalently equation (2.2)) driven by isotropic noise with spatial covariance given by (2.7) has a unique weak solution u such that $u(t, \cdot) \in L^{2}(D)$ for all $t \in \mathbb{R}_{+}$if and only if

$$
\sum_{l \in \mathbb{N}} \frac{a_{l}}{1+l}<\infty
$$


The proof of this theorem relies on Theorem 2.1 and precise estimates of the eigenvalues $\lambda_{n}$ and the eigenfunctions $e_{n}$, which both involve Bessel functions. In particular, rather recent estimates [4] on the behavior of zeros of Bessel functions are needed. A second key ingredient is a trace theorem for Sobolev spaces [2]. For complete details, the reader is referred to [10].

Condition (2.8) is explicit, because given a particular covariance $f$, we can directly compute the coefficients $a_{l}$ and check whether condition (2.8) is satisfied or not. For instance, a short calculation shows that the coefficients of white noise (which corresponds to $f(\cos \theta)=\delta_{0}(\theta)$ ) are of order

$$
a_{l} \underset{l \rightarrow \infty}{\sim} l^{d-2},
$$

so when $d \geq 2$, condition (2.8) is never satisfied and the solution $u$ is not $L^{2}(D)$-valued (recall that this is also the case of a space-time white noise spread over the whole domain $D($ see $[28])$ ).

In the case where $d=2$, the noise is concentrated on a circle. If we assume in this case that $f$ (viewed as a distribution on the circle) is non-negative (and therefore a measure), it can be checked [10] that condition (2.8) is equivalent to the following integral condition on $f$ :

$$
\sup _{\vartheta \in[-\pi, \pi]} \int_{-\pi}^{\pi} f(d \theta) \ln \left(\frac{\pi}{|\theta-\vartheta|}\right)<\infty .
$$

Notice that Theorem 2.3 does not tell us when the solution of the equation is a real-valued process $\left\{u(t, x),(t, x) \in \mathbb{R}_{+} \times D\right\}$. Indeed, an $L^{2}(D)$-valued function needs not necessarily be well defined at every point in $D$. We will obtain a precise answer concerning the difference between these two notions of solution in the following section, in the case of equations driven by noise on a hyperplane.

\section{Spatially homogeneous Gaussian noise on a hyperplane}

\subsection{The linear case}

In this section, we will often write an element $x \in \mathbb{R}^{d}$ as $x=\left(x_{1}, x_{2}\right) \in \mathbb{R}^{d-1} \times \mathbb{R}$, where $x_{1}$ denotes the first $d-1$ coordinates of $x$ and $x_{2}$ the last coordinate of $x$. We assume here that the boundary noise $\dot{F}$ is concentrated on the hyperplane $x_{2}=0$ and therefore we consider the following equation in $\mathbb{R}^{d}$ :

$$
\begin{cases}L u(t, x)=\dot{F}\left(t, x_{1}\right) \delta_{0}\left(x_{2}\right), & (t, x) \in \mathbb{R}_{+} \times \mathbb{R}^{d} \\ u(0, x)=\frac{\partial u}{\partial t}(0, x)=0, & x \in \mathbb{R}^{d}\end{cases}
$$

where $L$ is the operator defined in (1.2) and $\left\{\dot{F}\left(t, x_{1}\right),\left(t, x_{1}\right) \in \mathbb{R}_{+} \times \mathbb{R}^{d-1}\right\}$ is assumed to be a generalized centered Gaussian process with covariance

$$
\mathbb{E}\left(\dot{F}\left(t, x_{1}\right) \dot{F}\left(s, y_{1}\right)\right)=\delta_{0}(t-s) f\left(x_{1}-y_{1}\right),
$$

where $f$ is a non-negative definite distribution in the Schwartz space $\mathcal{S}\left(\mathbb{R}^{d-1}\right)$ of tempered distributions on $\mathbb{R}^{d-1}$.

As in Section 2, we can formally write the weak solution $u$ of equation (3.1) as

$$
u\left(t, x_{1}, x_{2}\right)=\int_{0}^{t} d s \int_{\mathbb{R}^{d-1}} d y_{1} \dot{F}\left(s, y_{1}\right) G\left(t-s, x_{1}-y_{1}, x_{2}\right), \quad\left(t, x_{1}, x_{2}\right) \in \mathbb{R}_{+} \times \mathbb{R}^{d-1} \times \mathbb{R},
$$

where $G$ is the Green kernel of the operator $L$ in $\mathbb{R}^{d}$. We would now like to establish an optimal condition on $f$ which guarantees that the solution $u$ of (3.1) is a real-valued process, indexed by $(t, x) \in \mathbb{R}_{+} \times \mathbb{R}^{d}$.

Let us respectively denote by $\mathcal{F} T, \mathcal{F}_{1} T$ and $\mathcal{F}_{2} T$ the Fourier transform of a distribution $T \in \mathcal{S}\left(\mathbb{R}^{d}\right)$, $\mathcal{S}\left(\mathbb{R}^{d-1}\right)$ or $\mathcal{S}(\mathbb{R})$. We will need the following Bochner-Schwartz theorem (see [26, Chap. VII, Thm XVIII]), analogous to Theorem 2.2 .

Theorem 3.1. $T \in \mathcal{S}\left(\mathbb{R}^{d-1}\right)$ is a non-negative definite distribution on $\mathbb{R}^{d-1}$ if and only if there exists a non-negative tempered measure $\mu$ on $\mathbb{R}^{d-1}$ such that $T=\mathcal{F}_{1} \mu$.

The measure $\mu$ is called the spectral measure of the noise $\dot{F}$; it is the continuous analog of the sequence $\left(a_{l}\right)$ of the preceding section.

We can now state the following theorem. 
Theorem 3.2. Equation (3.1) has a unique weak solution $u$ such that $\left\{u\left(t, x_{1}, x_{2}\right),\left(t, x_{1}, x_{2}\right) \in \mathbb{R}_{+} \times\right.$ $\left.\mathbb{R}^{d-1} \times \mathbb{R}^{*}\right\}$ is a real-valued process if and only if

$$
\int_{\mathbb{R}^{d-1}} \frac{\mu\left(d \xi_{1}\right)}{\sqrt{1+\left|\xi_{1}\right|^{2}}}<\infty
$$

Proof. We only sketch the main ideas. If $u\left(t, x_{1}, x_{2}\right)$ is a well-defined random variable, it is then certainly Gaussian, being the stochastic integral of a deterministic integrand with respect to a Gaussian process, therefore its variance must be finite. We compute this variance, using formally (3.3) together with (3.2):

$$
\begin{aligned}
\mathbb{E}\left(u\left(t, x_{1}, x_{2}\right)^{2}\right) & =\int_{0}^{t} d s \int_{\mathbb{R}^{d-1}} d y_{1} \int_{\mathbb{R}^{d-1}} d z_{1} G\left(t-s, x_{1}-y_{1}, x_{2}\right) f\left(y_{1}-z_{1}\right) G\left(t-s, x_{1}-z_{1}, x_{2}\right) \\
& =\int_{0}^{t} d s \int_{\mathbb{R}^{d-1}} \mu\left(d \xi_{1}\right) \mathcal{F}_{1} G\left(s, \xi_{1}, x_{2}\right)^{2}
\end{aligned}
$$

by Theorem 3.1. Assume for simplicity that $a=b=0$. In order to compute $\mathcal{F}_{1} G\left(s, \xi_{1}, x_{2}\right)$, let us first note that for any dimension $d \geq 1$, we have the following expression for the Fourier transform of the Green kernel in $\mathbb{R}^{d}$ :

$$
\mathcal{F} G(s, \xi)=\frac{\sin (s|\xi|)}{|\xi|}, \quad(s, \xi) \in \mathbb{R}_{+} \times \mathbb{R}^{d}
$$

So by the Fourier inversion formula and formula I.5.83 in [20], we have

$$
\mathcal{F}_{1} G\left(s, \xi_{1}, x_{2}\right)=\mathcal{F}_{2}^{-1}\left(\mathcal{F} G\left(s, \xi_{1}, \cdot\right)\right)\left(x_{2}\right)=\frac{1}{2} J_{0}\left(\left|\xi_{1}\right| \sqrt{s^{2}-x_{2}^{2}}\right) 1_{\left\{\left|x_{2}\right|<s\right\}},
$$

where $J_{0}$ is the zero-order regular Bessel function. Using standard estimates on $J_{0}$, it is possible to show that (3.5) is finite for all $x_{2} \neq 0$ if and only if condition (3.4) is satisfied. For complete details, see [11].

Theorem 3.2 provides a different type of statement than Theorem 2.3, since it gives the existence of a real-valued random field $\left\{u\left(t, x_{1}, x_{2}\right)\right\}$ rather than the existence of an $L(D)$-valued random process $\{u(t, \cdot)\}$. However, under condition $(3.4)$, the random field $\left\{u\left(t, x_{1}, x_{2}\right)\right\}$ need not be well defined on the hyperplane $x_{2}=0$. For this, a slightly stronger condition is needed, as the following theorem shows.

Theorem 3.3. Equation (3.1) has a unique weak solution u such that $\left\{u(t, x),(t, x) \in \mathbb{R}_{+} \times \mathbb{R}^{d}\right\}$ is a real-valued process if and only if

$$
\int_{\mathbb{R}^{d-1}} \frac{\mu\left(d \xi_{1}\right) \ln \left(1+\left|\xi_{1}\right|^{2}\right)}{\sqrt{1+\left|\xi_{1}\right|^{2}}}<\infty
$$

The proof of this theorem is also based on precise estimates of the Bessel function $J_{0}$. For complete details, the reader is referred to [11].

Conditions (3.4) and (3.6) are both necessary and sufficient conditions and, of course, there exist noises $\dot{F}$ with a spectral measure $\mu$ that satisfies (3.4), but not (3.6). Note that white noise, which has spectral measure $\delta_{0}$, does not satisfy either of the two conditions (3.4) or (3.6) when $d \geq 2$, as was the case for white noise on the sphere and condition (2.8) in the preceding section.

If $f$ is non-negative (and therefore a measure on $\mathbb{R}^{d-1}$ ), then condition (3.4) can be expressed as an explicit condition on $f$. Indeed, (3.4) implies (and is "nearly implied" [11] by)

$$
\left\{\begin{array}{l}
\int_{\left|x_{1}\right|<1} f\left(d x_{1}\right) \ln \left(\frac{1}{\left|x_{1}\right|}\right)<\infty, \quad \text { when } d=2, \\
\int_{\left|x_{1}\right|<1} f\left(d x_{1}\right) \frac{1}{\left|x_{1}\right|^{d-2}}<\infty, \quad \text { when } d \geq 3 .
\end{array}\right.
$$

Observe the similarity of the case $d=2$ with condition (2.9) for a noise on a circle.

Under a slightly stronger condition than (3.6), it is possible to establish some regularity properties of the solution $u$. This is the object of the following theorem.

Theorem 3.4. Let $\beta \in] 0, \frac{1}{2}[$. If

$$
\int_{\mathbb{R}^{d-1}} \frac{\mu\left(d \xi_{1}\right)}{\left(1+\left|\xi_{1}\right|^{2}\right)^{\frac{1}{2}-\beta}}<\infty
$$

then the process $u$ solution of equation (3.1) is a.s. locally Hölder-continuous with exponent $\gamma<\beta \wedge \frac{1}{4}$ outside the hyperplane $x_{2}=0$. 
The proof of this theorem relies on the Kolmogorov continuity theorem and on precise estimates of the variance of the increments of the process $u$; it is related to the estimates of [24] concerning s.p.d.e.'s driven by spatially homogeneous noises spread over the whole space. Complete details are given in [11].

\subsection{The non-linear case}

With the results of Section 3.1, it is now possible to consider the following non-linear equation, which was one of the objectives mentioned in the introduction:

$$
\begin{cases}L u(t, x)=\left(\alpha\left(u\left(t, x_{1}, 0\right)\right)+\beta\left(u\left(t, x_{1}, 0\right)\right) \dot{F}\left(t, x_{1}\right)\right) \delta_{0}\left(x_{2}\right), & (t, x) \in \mathbb{R}_{+} \times \mathbb{R}^{d}, \\ u(0, x)=\frac{\partial u}{\partial t}(0, x)=0, & x \in \mathbb{R}^{d},\end{cases}
$$

where $\alpha, \beta$ are Lipschitz-continuous functions on $\mathbb{R}$. When $d \leq 3$ and the covariance $f$ is non-negative, a rigorous interpretation of this equation is given by its mild formulation:

$$
u\left(t, x_{1}, x_{2}\right)=\int_{0}^{t} d s \int_{\mathbb{R}^{d-1}} d y_{1}\left(\alpha\left(u\left(s, y_{1}, 0\right)\right)+\beta\left(u\left(s, y_{1}, 0\right)\right) \dot{F}\left(s, y_{1}\right)\right) G\left(t-s, x_{1}-y_{1}, x_{2}\right) .
$$

The assumptions on $d$ and $f$ are needed to give a rigorous meaning to the stochastic integral on the right-hand side, which is an extension of the martingale-measure stochastic integral, as developed in $[8,16]$.

Theorem 3.5. Under condition (3.6) and the above assumptions, there exists a unique random field $\left\{u(t, x),(t, x) \in \mathbb{R}_{+} \times \mathbb{R}^{d}\right\}$ which is a mild solution of (3.9).

Proof. The key idea is to consider first the restriction of equation (3.10) to the hyperplane $x_{2}=0$, and to show, using Picard's iteration scheme as in $[8,9]$, that there is a unique solution of this restricted equation. Then formula (3.10) defines the solution $u(t, x)$ for $x_{2} \neq 0$ and all $t>0$. Full details are provided in $[11]$.

\section{References}

[1] Adler R.J., Müller P., Rozovskii B.L., "Stochastic modelling in physical oceanography", Prog. in Prob., Vol. 39, 1996, Birkhäuser.

[2] Adams R.A., "Sobolev spaces", 1975, Academic Press.

[3] Brace A., Gatarek D., Musiela M., "The market model of interest rate dynamics", Math. Finance, Vol. 7 (2), 1997, pp. 127-155.

[4] Breen S., "Uniform upper and lower bounds on the zeros of Bessel functions of the first kind", J. Math. Anal. Appl., Vol. 196 (1), 1995, pp. 1-17.

[5] Carmona R.A., Rozovskii B.L., "Stochastic partial differential equations: six perspectives", Mathematical Surveys and Monographs Nr 64, 1999, American Mathematical Society.

[6] Duan J., Schmalfuss B., "The 3D quasigeostrophic fluid dynamics under random forcing on boundary", preprint.

[7] Dalang R. C., Frangos N. E., "The stochastic wave equation in two spatial dimensions", Ann. Prob., Vol. 26 (1), 1998, pp. 187-212.

[8] Dalang R.C., "Extending the martingale measure stochastic integral with applications to spatially homogeneous SPDE's", Electronic Journal of Probability, Vol. 4, 1999, Article Nr 6.

[9] Dalang R.C., "Corrections to Extending the martingale measure stochastic integral with applications to spatially homogeneous SPDE's", Electronic Journal of Probability, Vol. 6, 2001.

[10] Dalang R.C., Lévêque O., "Second order linear hyperbolic SPDE's driven by isotropic Gaussian noise on a sphere", preprint.

[11] Dalang R.C., Lévêque O., "Second order hyperbolic SPDE's driven by homogeneous Gaussian noise on a hyperplane", in preparation.

[12] Da Prato G., Zabczyk J., "Evolution equations with white-noise boundary conditions", Stoch. and Stoch. Reports, Vol. 42, 1993, pp. 167-182.

[13] Da Prato G., Zabczyk J., "Stochastic equations in infinite dimensions", Encyclopedia of Mathematics and its Applications 44, 1992, Cambridge University Press.

[14] Da Prato G., Zabczyk J., "Ergodicity for infinite-dimensional systems", London Mathematical Society Lecture Note Series 229, 1996, Cambridge University Press. 
[15] Karczewska A., Zabczyk J., "Stochastic PDE's with function-valued solutions", in Clément Ph., den Hollander F., van Neerven J. and de Pagter B. (Eds), "Infinite-dimensional stochastic analysis", Proceedings of the Colloquium of the Royal Netherlands Academy of Arts and Sciences, 1999, Amsterdam.

[16] Lévêque O., "Hyperbolic SPDE's driven by boundary noises", PhD Thesis No. 2452 (2001), EPF Lausanne.

[17] Mao X., Markus L., "Wave equation with stochastic boundary values", J. Math. Anal. and Appl., Vol. 177, 1993, pp. 315-341.

[18] Maslowski B., "Stability of semilinear equations with boundary and pointwise noise", Annali della Scuola Normale Superiore di Pisa, Serie IV, Vol. XXII, 1995, Fasc. 1.

[19] Millet A., Sanz-Solé M. "A stochastic wave equation in two space dimension: Smoothness of the law", Annals Probab., Vol. 27 (2), 1999, pp. 803-844.

[20] Oberhettinger F., "Tables of Fourier transforms and Fourier transforms of distributions", 1990, Springer Verlag.

[21] Peszat S., "The Cauchy problem for a nonlinear stochastic wave equation in any dimension", preprint.

[22] Peszat S., Zabczyk J., "Stochastic evolution equations with a spatially homogeneous Wiener process", Stoch. Proc. and Appl., Vol. 72, 1997, pp. 187-204.

[23] Peszat S., Zabczyk J., "Nonlinear stochastic wave and heat equations", Prob. Th. and Rel. Fields, Vol. 116, 2000, pp. 421-443.

[24] Sanz-Solé M., Sarrà M., "Path properties of a class of Gaussian processes with applications to SPDE's", in Gestesy F., Holde H., Jost H., Paycha S., Röckner M. and Scarlatti S. (Eds), "Stochastic porcesses, physics and geometry: new interplays I (Leipzig, 1999)", CMS Conference Proceedings, Vol. 28, 2000, Providence(RI), pp. 303-316.

[25] Schönberg I.J., "Positive definite functions on spheres", Duke Mathematical Journal, Vol. 9, 1942, pp. 96-108.

[26] Schwartz L., "Théorie des distributions", 1966, Hermann.

[27] Sowers R., "Multidimensional reaction-diffusion equations with white noise boundary perturbations", Ann. of Prob., Vol. 22 (4), 1994, pp. 2071-2121.

[28] Walsh J.B., "An introduction to stochastic partial differential equations", Ecole d'été de probabilités de Saint-Flour XIV, Lecture Notes in Math., 1984, Springer Verlag.

Institut de Mathémathiques, Faculté des Sciences de Base, Ecole Polytechnique Fédérale de Lausanne, CH - 1015 Lausanne

E-mail address: robert.dalang@epfl.ch

Laboratoire de Théorie de l'Information, Institut de Systèmes de Communications, Faculté Informatique et Communications, Ecole Polytechnique Fédérale de Lausanne, CH - 1015 Lausanne

E-mail address: olivier.leveque@epfl.ch 